\title{
ON BLEIMANN-BUTZER-HAHN OPERATORS FOR EXPONENTIAL FUNCTIONS
}

\section{UlRICH ABEl AND Mircea IVAN}

Some inequalities involving the binomial coefficients are obtained. They are used to determine the domain of convergence of the Bleimann, Butzer and Hahn approximation process for exponential type functions. An answer to Hermann's conjecture related to the Bleimann, Butzer and Hahn operators for monotone functions is given.

\section{INTRODUCTION}

In 1980, Bleimann, Butzer and Hahn [2], introduced the linear operators $L_{n}$ defined by

$$
L_{n}(f, x):=\frac{1}{(1+x)^{n}} \sum_{k=0}^{n}\left(\begin{array}{l}
n \\
k
\end{array}\right) x^{k} f\left(\frac{k}{n+1-k}\right), x \in[0, \infty), n=0,1, \ldots,
$$

in order to approximate continuous functions $f:[0, \infty) \rightarrow \mathbb{R}$ on the positive semi-axis. The operators $L_{n}$ were investigated by several authors (see References).

Totik [8] studied the uniform approximation properties of these operators when $f$ belongs to the class $C[0, \infty]$ of continuous functions on $[0, \infty)$ that have finite limits at infinity.

Jayasri and Sitaraman [4] considered the class

$$
C_{P_{N}}[0, \infty):=\left\{f \in C[0, \infty): f(x)=O\left((1+x)^{N}\right)(x \rightarrow+\infty)\right\},
$$

where $N$ is a fixed positive integer. They proved that if $f$ belongs to $C_{P_{N}}[0, \infty)$, then for each $x \geqslant 0, \lim _{n \rightarrow \infty} L_{n}(f ; x)=f(x)[4$, Theorem 2.1].

Hermann [3] defined a function class $\mathcal{H}$ in the following way

$$
\mathcal{H}:=\{f \in C[0, \infty): \log (|f(x)|+1)=o(x) \text { as } x \rightarrow+\infty\} .
$$

Hermann proved that if $f$ belongs to $\mathcal{H}$ then, for each $x \geqslant 0, \lim _{n \rightarrow \infty} L_{n}(f ; x)=f(x)$. Moreover, if for some $\alpha>0, f(x)=e^{\alpha x}$, then $\lim _{n \rightarrow \infty} L_{n}(f ; x)=\infty$, provided that $x$ is sufficiently large [3, Theorem 1]. He also stated the following

Received 31st October, 2006

The authors would like to thank Thomas Hermann for his useful comments on the manuscript. Furthermore, they are grateful to the referee, whose valuable hints have led to a better presentation of the paper.

Copyright Clearance Centre, Inc. Serial-fee code: 0004-9727/07 \$A2.00+0.00. 
Conjecture 1.1. (Hermann, 1990, [3]) If $f \in C[0, \infty)$ and $L_{n} f$ converges pointwise to $f$ on $[0, \infty)$, then $f \in \mathcal{H}$.

Jayasri and Sitaraman [5] introduced the function class

$$
\mathcal{F}:=\left\{f \in C[0, \infty): \text { for each } A>0, f(x)=O\left(e^{A x}\right) \text { as } x \rightarrow+\infty\right\} .
$$

They proved that $\left(L_{n}\right)$ defines a pointwise approximation process on $\mathcal{F}$ [5, Theorem 2.1]. Moreover, if $f(x)=e^{A x}$, for some $A>0$, then $\lim _{n \rightarrow \infty} L_{n}(f ; x)=\infty$, provided that $x$ is sufficiently large [5, Remark, p. 270].

A key result of Jayasri and Sitaraman is the inequality

$$
\sum_{|k /(n-k)-x| \geqslant \delta} p_{n, k}\left(\frac{x}{1+x}\right) \leqslant 2 \exp \left(-\frac{n \delta^{2}}{16 x(1+x)^{2}}\right),
$$

$0<\delta \leqslant x, n \in \mathbb{N}\left[5\right.$, Lemma 2.3], where $p_{n, k}(t)=\left(\begin{array}{l}n \\ k\end{array}\right) t^{k}(1-t)^{n-k}$ are the Bernstein basis polynomials. It was derived from Bernstein's inequality [7, p. 18, Equation (20)]

$$
\sum_{|k-n t| \geqslant 2 z(n t(1-t))^{1 / 2}} p_{n, k}(t) \leqslant 2 \exp \left(-z^{2}\right),
$$

by using the transform $t=x /(1+x)$, for $0 \leqslant t \leqslant 1,0 \leqslant z \leqslant(3 / 2)(n t(1-t))^{1 / 2}$.

Hermann [3] proved that for any $x \geqslant 0$ there exists $q(x)>0$ such that

$$
\sum_{(n+1)((x+1) /(x+2)) \leqslant k \leqslant n} p_{n, k}\left(\frac{x}{1+x}\right)=O(\exp (-n q(x))),
$$

provided that $n$ is sufficiently large.

We shall prove that for all $x \geqslant 0$ and $\varepsilon>0$ there exists $\theta \in(0,1)$ such that

$$
\sum_{k=\lfloor\theta n]}^{n} p_{n, k}\left(\frac{x}{1+x}\right) \leqslant\left(\frac{x}{1+x}+\varepsilon\right)^{n}
$$

provided that $n$ is sufficiently large.

Finding the domain of convergence of the Bleimann, Butzer and Hahn approximation process is a difficult task, not yet solved.

We use our inequality (7) to determine the domain of convergence of the sequence of Bleimann, Butzer and Hahn operators for exponential type functions. Moreover we deduce that $f \in \mathcal{H}$ is a sufficient condition for the pointwise convergence of the sequence $\left(L_{n} f\right)$ to $f$. Finally we show that Hermann's conjecture is true for continuous monotone functions. 


\section{Preliminary Results}

Suppose that $x>0$ and $n$ be an integer.

LEMMA 2.1. The following inequality

$$
\sum_{k=p}^{n}\left(\begin{array}{l}
n \\
k
\end{array}\right) x^{k} \leqslant 2\left(\begin{array}{l}
n \\
p
\end{array}\right) x^{p}
$$

is valid for $(2 x /(2 x+1)) n \leqslant p \leqslant n$.

Proof: Suppose that $p, n$ be integers satisfying $1 \leqslant p \leqslant n$. Our starting point is the known equality

$$
\sum_{k=p}^{n}\left(\begin{array}{l}
n \\
k
\end{array}\right) x^{k}=(1+x)^{n} \frac{B(p, n+1-p,(x /(1+x)))}{B(p, n+1-p)}
$$

(see, for example, [6, Equation (16), p. 86]) with the incomplete Beta-function

$$
B(a, b, u)=\int_{0}^{u} t^{a-1}(1-t)^{b-1} d t \quad(a, b>0,0 \leqslant u \leqslant 1) .
$$

Using the representation

$$
B\left(a, b, \frac{x}{1+x}\right)=\int_{0}^{x} \frac{t^{a-1}}{(1+t)^{a+b}} d t
$$

one can easily obtain:

$$
B\left(p+1, n-p, \frac{x}{1+x}\right) \leqslant x B\left(p, n+1-p, \frac{x}{1+x}\right)
$$

and

$$
B\left(p, n+1-p, \frac{x}{1+x}\right)=\frac{x^{p}}{p(1+x)^{n}}+\frac{n-p}{p} B\left(p+1, n-p, \frac{x}{1+x}\right)
$$

From Eqs. (9) and (10) we conclude that

$$
B\left(p, n+1-p, \frac{x}{1+x}\right) \leqslant 2 \frac{x^{p}}{p(1+x)^{n}} \quad\left(p \geqslant n \frac{2 x}{1+2 x}\right) .
$$

Inserting (11) into (8) completes the proof.

LEMMA 2.2. The following inequality

$$
\left(\begin{array}{l}
n \\
p
\end{array}\right)<\frac{1}{2} \frac{n^{n}}{p^{p}(n-p)^{n-p}}
$$

is valid for $n>2$ and $p=1, \ldots, n-1$. 
ProOF: By Stirling's Formula $[1,6.1 .38]$

$$
z !=\sqrt{2 \pi} z^{z} \sqrt{z} \exp \left(-z+\frac{\lambda}{12 z}\right) \quad(z>0, \lambda \in(0,1))
$$

with $p=\lfloor\theta n\rfloor$, we obtain

$$
\left(\begin{array}{l}
n \\
p
\end{array}\right)=\frac{1}{\sqrt{2 \pi}} \sqrt{\frac{n}{p(n-p)}} \frac{n^{n}}{p^{p}(n-p)^{n-p}}(1+o(1)),
$$

hence

$$
\left(\begin{array}{l}
n \\
p
\end{array}\right)<\frac{1}{2} \frac{n^{n}}{p^{p}(n-p)^{n-p}}
$$

provided that $n$ is sufficiently large.

LEmma 2.3. Suppose that $x \in[0, \infty)$ is fixed. For any $\varepsilon>0$ there exists $\theta \in(0,1)$ such that

$$
\sum_{k=\lfloor\theta n\rfloor}^{n}\left(\begin{array}{l}
n \\
k
\end{array}\right) \frac{x^{k}}{(1+x)^{n}} \leqslant\left(\frac{x}{1+x}+\varepsilon\right)^{n},
$$

provided that $n$ is sufficiently large.

PROOF: For fixed $x \in[0, \infty)$, we define the function

$$
g_{x}(t)=\frac{(x /(1+x))^{t}(1-(x /(1+x)))^{1-t}}{t^{t}(1-t)^{1-t}}, \quad t \in(0,1) .
$$

It follows from the equality

$$
\lim _{t \nearrow 1} g_{x}(t)=\frac{x}{1+x}
$$

that for any $\varepsilon>0$ there exists $\theta \in(2 x /(2 x+1), 1)$ such that

$$
g_{x}\left(\frac{\lfloor\theta n\rfloor}{n}\right)<\frac{x}{1+x}+\varepsilon
$$

provided that $n$ is sufficiently large. Application of Lemmata 2.1 and 2.2, with $p=\lfloor\theta n\rfloor$ yields

$$
\begin{aligned}
\sum_{k=p}^{n}\left(\begin{array}{l}
n \\
k
\end{array}\right) \frac{x^{k}}{(1+x)^{n}} \leqslant & 2\left(\begin{array}{l}
n \\
p
\end{array}\right) \frac{x^{p}}{(1+x)^{n}}<\frac{n^{n}}{p^{p}(n-p)^{n-p}} \frac{x^{p}}{(1+x)^{n}} \\
& \left(\frac{(x /(1+x))^{p / n}(1-(x /(1+x)))^{1-p / n}}{(p / n)^{p / n}(1-(p / n))^{1-(p / n)}}\right)^{n}=\left(g_{x}\left(\frac{p}{n}\right)\right)^{n} .
\end{aligned}
$$

and the proof is completed by applying inequality (12). 
3. THE BEHAVIOUR OF THE SEQUENCE $\left(L_{n}\right)$ FOR EXPONENTIAL FUNCTIONS.

The following theorem describes the exact domain of convergence of the sequence of operators of Bleimann, Butzer and Hahn for exponential functions. It is also an improvement on the result reported by Hermann in [3, Theorem 1(ii)].

THEOREM 3.1. For each $a>1$, there holds

$$
\lim _{n \rightarrow \infty} L_{n}\left(a^{t} ; x\right)=a^{x} \quad \text { if and only if } \quad x \in\left[0, \frac{1}{a-1}\right) .
$$

Proof: If $x \in[0,1 /(a-1))$ then $a(x /(1+x))<1$. It follows that there exists $\varepsilon>0$ such that

$$
a\left(\frac{x}{1+x}+\varepsilon\right)<1 .
$$

By Lemma 2.3 there exists $\theta \in(0,1)$ such that

$$
\sum_{k=\lfloor\theta n\rfloor}^{n}\left(\begin{array}{l}
n \\
k
\end{array}\right) \frac{x^{k}}{(1+x)^{n}} \leqslant\left(\frac{x}{1+x}+\varepsilon\right)^{n},
$$

provided that $n$ is sufficiently large. Obviously, we can assume that $\theta>2 x /(1+2 x)$, which is equivalent to $\theta /(1-\theta)>2 x$.

Following Hermann [3], we define the function $\varphi:[0, \infty) \rightarrow \mathbb{R}$,

$$
\varphi(t)= \begin{cases}1, & 0 \leqslant t \leqslant \frac{\theta}{1-\theta}, \\ 0, & t>\frac{\theta}{1-\theta} .\end{cases}
$$

We have

$$
L_{n}\left(a^{t} ; x\right)=L_{n}\left(\varphi(t) \cdot a^{t} ; x\right)+L_{n}\left((1-\varphi(t)) \cdot a^{t} ; x\right) .
$$

The pointwise convergence of the sequence $\left(L_{n} f\right)$ for any continuous bounded function $f$ is well known. Since the function $t \mapsto \varphi(t) \cdot a^{t}$ is bounded and continuous in $x$, we deduce

$$
\lim _{n \rightarrow \infty} L_{n}\left(\varphi(t) \cdot a^{t} ; x\right)=\varphi(x) \cdot a^{x}=a^{x} .
$$

Finally, we shall show that $L_{n}\left((1-\varphi(t)) \cdot a^{t} ; x\right)$ tends to zero as $n \rightarrow \infty$. We have

$$
\begin{aligned}
\left|L_{n}\left((1-\varphi(t)) \cdot a^{t} ; x\right)\right| & =\sum_{k /(n+1-k) \geqslant \theta /(1-\theta)}\left(\begin{array}{l}
n \\
k
\end{array}\right) \frac{x^{k}}{(1+x)^{n}} a^{k /(n+1-k)} \\
& =\sum_{k \geqslant(n+1) \theta}\left(\begin{array}{l}
n \\
k
\end{array}\right) \frac{x^{k}}{(1+x)^{n}} a^{k /(n+1-k)} \leqslant a^{n} \sum_{k \geqslant(n+1) \theta}\left(\begin{array}{l}
n \\
k
\end{array}\right) \frac{x^{k}}{(1+x)^{n}} \\
& \leqslant a^{n}\left(\frac{x}{1+x}+\varepsilon\right)^{n} \rightarrow 0,
\end{aligned}
$$


for $n \rightarrow \infty$ (we applied (13)). In the case of $x \geqslant 1 /(a-1)$, we obtain

$$
L_{n}\left((1-\varphi(t)) \cdot a^{t} ; x\right)>a^{n}\left(\frac{x}{1+x}\right)^{n} \geqslant 1 .
$$

Hence, $L_{n}\left((1-\varphi(t)) \cdot a^{t} ; x\right)$ does not tend to zero as $n \rightarrow \infty$.

The following theorem follows immediately from Theorem 3.1.

Theorem 3.2. Suppose that $a>1$. If $f \in C[0, \infty)$ satisfies $K_{1} a^{x} \leqslant f(x)$ $\leqslant K_{2} a^{x}$, for all $x \in(0, \infty)$, with constants $K_{1}, K_{2}>0$, then

$$
\lim _{n \rightarrow \infty} L_{n}(f ; x)=f(x) \text { if and only if } x \in\left[0, \frac{1}{a-1}\right) .
$$

It is easy to show that the class $\mathcal{H}$ defined by Equation (2) can be written in the form

$$
\mathcal{H}=\left\{f \in C[0, \infty): \lim _{x \rightarrow+\infty} \frac{f(x)}{a^{x}}=0, \quad \forall a>1\right\} .
$$

Hence, as a consequence of Theorem 3.1 we obtain

THEOREM 3.3. If $f \in \mathcal{H}$, then $L_{n} f$ converges pointwise to $f$ on $[0, \infty)$.

Theorem 3.3 is similar to [3, Theorem 1(i)].

\section{A partial solution to Hermann's conjecture}

In this section we give an answer to Hermann's conjecture for the case of monotone functions.

THEOREM 4.1. If $f \in C[0, \infty)$ is monotone and $L_{n} f$ converges pointwise to $f$ on $[0, \infty)$, then $f \in \mathcal{H}$.

Proof: For definiteness, we assume that $f$ is a non-decreasing function such that $f \geqslant 0$ (if that is not the case, then we consider $f-f(0)$ instead of $f$ ). Suppose that $f$ does not belong to the class $\mathcal{H}$. It follows that there exist $a>1, M>0$, and a sequence of positive numbers $\left(t_{n}\right)$ tending to infinity, such that

$$
\frac{f\left(t_{n}\right)}{a^{t_{n}}} \geqslant M, \text { for all } n \in \mathbb{N} .
$$

For $m=\left\lfloor t_{n}\right\rfloor+1$ and $x>1 /(a-1)$, we obtain

$$
L_{m}(f ; x) \geqslant f(m)\left(\frac{x}{1+x}\right)^{m} \geqslant M a^{m-1}\left(\frac{x}{1+x}\right)^{m} \rightarrow \infty \quad(m \rightarrow \infty) .
$$

This contradicts the assumption $\lim _{m \rightarrow \infty} L_{m}(f ; x)=f(x)$, and the proof is completed. 


\section{REFERENCES}

[1] M. Abramowitz and I.A. Stegun, Handbook of Mathematical Functions, (ninth printing) (Dover, 1970).

[2] G. Bleimann, P.L. Butzer, and L. Hahn, 'A Bernšteĭn-type operator approximating continuous functions on the semi-axis', Nederl. Akad. Wetensch. Indag. Math. 42 (1980), 255-262.

[3] T. Hermann, 'On the operator of Bleimann, Butzer and Hahn', in Approximation theory (Kecskemét, 1990), Colloq. Math. Soc. János Bolyai 58 (North-Holland, Amsterdam, 1991), pp. 355-360,.

[4] C. Jayasri and Y. Sitaraman, 'Direct and inverse theorems for certain Bernstein-type operators', Indian J. Pure Appl. Math. 16 (1985), 1495-1511.

[5] C. Jayasri and Y. Sitaraman, 'On a Bernstein-type operator of Bleimann, Butzer and Hahn', J. Comput. Appl. Math. 47 (1993), 267-272.

[6] C. Jordan, Calculus of finite differences (Röttig and Romwalter, Budapest, 1939).

[7] G.G. Lorentz, Bernstein polynomials (Univ. Toronto Press, Toronto, 1953).

[8] V. Totik, 'Uniform approximation by Bernstein-type operators', Nederl. Akad. Wetensch. Indag. Math. 46 (1984), 87-93.

Fachhochschule Giessen-Friedberg

University of Applied Sciences

Fachbereich MND

61169 Friedberg

Germany
Department of Mathematics

Technical University of Cluj-Napoca 400020 Cluj-Napoca

Romania 